\title{
ВMJ Global Health Contextual equipoise: a novel concept to inform ethical implications for implementation research in low-income and middle-income countries
}

\author{
Nadine Seward (D) , ${ }^{1}$ Charlotte Hanlon (D) , 2,3,4 Jamie Murdoch (D) , \\ Tim Colbourn (D) , ${ }^{6}$ Martin James Prince (D) , Sridhar Venkatapuram (D) , ${ }^{4}$ \\ Nick Sevdalis ${ }^{4}$
}

To cite: Seward N, Hanlon C, Murdoch J, et al. Contextual equipoise: a novel concept to inform ethical implications for implementation research in low-income and middle-income countries. BMJ Global Health 2020;5:e003456. doi:10.1136/ bmjgh-2020-003456

Handling editor Seye Abimbola TC and MJP contributed equally.

Received 15 July 2020 Revised 6 October 2020 Accepted 8 October 2020

Check for updates

(c) Author(s) (or their employer(s)) 2020. Re-use permitted under CC BY-NC. No commercial re-use. See rights and permissions. Published by BMJ.

For numbered affiliations see end of article.

Correspondence to Dr Nadine Seward; nadine.seward@kcl.ac.uk

\section{ABSTRACT}

The call for universal health coverage requires the urgent implementation and scale-up of interventions that are known to be effective, in resource-poor settings. Achieving this objective requires high-quality implementation research (IR) that evaluates the complex phenomenon of the influence of context on the ability to effectively deliver evidence-based practice. Nevertheless, IR for global health is failing to apply a robust, theoretically driven approach, leading to ethical concerns associated with research that is not methodologically sound.

Inappropriate methods are often used in IR to address and report on context. This may result in a lack in understanding of how to effectively adapt the intervention to the new setting and a lack of clarity in conceptualising whether there is sufficient evidence to generalise findings from previous $I R$ to a new setting, or if a randomised controlled trial (RCT) is needed. Some of the ethical issues arising from this shortcoming include poor-quality research that may needlessly expose vulnerable participants to research that has not been adapted to suit local needs and priorities, and the inappropriate use of RCTs that denies participants in the control arm access to treatment that is effective within the local context.

To address these concerns, we propose a complementary approach to clinical equipoise for IR, known as contextual equipoise. We discuss challenges in the evaluation of context and also with assessing the certainty of evidence to justify an RCT. Finally, we describe methods that can be applied to improve the evaluation and reporting of context and to help understand if contextual equipoise can be justified or if significant adaptations are required. We hope our analysis offers helpful insight to better understand and ensure that the ethical principle of beneficence is upheld in the real-world contexts of IR in low-resource settings.

\section{BACKGROUND}

The launch of the sustainable development goals (SDGs) and the call for universal health coverage with high-quality care marks a new era in the global health agenda. ${ }^{1}$ Achieving these goals will require implementation
Summary box

- Implementation research (IR) involves a theorydriven approach to understand how context influences the effectiveness of implementation strategies and the evidence-based practices they deliver in real-world settings.

- However, IR in low-resource settings that inadequately evaluates context raises ethical concerns about the impact of that research on the communities it is intended to benefit.

- To address these concerns we propose the concept of contextual equipoise as a criterion for justifying $\mathbb{I R}$, defined as 'genuine uncertainty as to whether the implementation strategies will effectively deliver the evidence-based practice in a new context'.

- We describe robust, evidence-based theories and methods for assessing contextual equipoise to determine if an intervention requires minor adaptations for scale-up, or whether a randomised controlled trial is necessary and justified.

- Assessing contextual equipoise can ensure the ethical principle of beneficence is upheld by ensuring participants in the control arm of a trial are not denied access to care that is known to be effective in the local context, and the intervention responds to the needs and priorities of the community.

- Policy relevant recommendations include development of ethical guidelines that specify standards for IR including how to evaluate and report context.

- However, existence of guidelines alone will not necessarily ensure that research ethics committees adhere to recommendations, where including ethical guidelines in the accreditation of ethics committees may help to overcome this issue.

research (IR) that uses a robust, theoryinformed approach to test innovative implementation strategies to deliver evidencebased practices (EBPs) at scale in resourcepoor contexts within low/middle-income countries (LMICs). ${ }^{2}$ However, if these goals 
are to be realised, key ethical challenges will need to be addressed, including those associated with IR that is not methodologically sound.

A particular area of concern is ensuring that context is adequately assessed so that the intervention is adapted to respond to the needs and priorities of the local population. Although a consideration of context applies to all forms of IR design, this has particular pertinence in the design of randomised controlled trials (RCTs), to ensure that participants in the control arm are not exposed to unnecessary harms associated with denying individuals access to care that is known or can be expected to be effective in the local context. This is especially relevant when access to high-quality care is limited, or in some instances non-existent. ${ }^{3}$

We provide a brief overview of IR and describe some of the associated limitations with this research in LMICs. We review the importance of addressing context and the distinction between clinical and contextual equipoise. We also discuss challenges in conceptualising contextual uncertainty and propose theoretical and methodological approaches for determining contextual equipoise. Finally, we conclude by proposing an operational definition of contextual equipoise for IR in LMICs.

\section{OVERVIEW OF IR}

IR is a rapidly expanding discipline that applies a multidisciplinary, theory-informed approach to understand what, why and how implementation strategies and the EBP they deliver work in real-world setting. ${ }^{4}$ In the preimplementation phase of research, investigators typically use a mixed-methods approach to evaluate the local context to determine what the barriers (and drivers) of implementation may be, so that they can subsequently select and adapt appropriate implementation strategies (eg, task shifting using community health workers) to address barriers and support delivery of the EBP. ${ }^{56}$ Throughout the implementation phase, researchers also monitor and evaluate the influence of context on effectiveness of the selected implementation strategies in delivering EBP in terms of the outcomes of the implementation process (ie, the acceptability, coverage and sustainability that the implementation achieves). ${ }^{4}$

Although IR using robust methods is common within high-income countries, more often than not, this does not apply to IR in LMICs. The scarcity of published literature describing high quality IR using appropriate methodology, exposes a lack of high quality research in LMICs. ${ }^{78}$ This is despite the availability of peerreviewed articles describing the importance of a robust, theoretically informed approach to IR in LMICs. ${ }^{7-11}$ As an example, frequently IR in LMICs is not reported or described as such; or the fact that a study is testing the effectiveness of implementation strategies to deliver EBP in the LMIC context is sidelined. ${ }^{9}$ A review of IR in LMICS also found that only 791 (8\%) out of 10292 published, peer reviewed IR articles describe the evidence-based interventions and set of implementation strategies they used to deliver them. ${ }^{9}$ Importantly, only a few publications reported use of a programme theory. The current dearth of evidence supporting the effectiveness of implementation strategies in LMICs is thus unsurprising - and remains problematic. ${ }^{12}$

Although global health researchers are becoming increasingly interested in understanding why some interventions are effective while others fail, very often these researchers focus on quantifying the impact of the interventions, instead of using a mixed-methods approach to uncover the complexity surrounding what works for whom and how. ${ }^{7}$ A result of this focus on clinical effectiveness is that IR in LMICs is failing to adequately address context. Of the 791 articles in the previously described review that reported their research as IR, only $52 \%(n=415)$ described contextual determinants. ${ }^{9}$ Furthermore, the IR that has reported on context tends to focus on contextual determinants that are external to the healthcare facility (ie, sociocultural and political determinants-ie, outer context), leaving a gap in the evidence-base for the influence of contextual determinants at the health facility level (ie, inner context) on implementation effectiveness. ${ }^{9}$ A result of IR in LMICs failing to address context adequately is that findings cannot be easily generalised and replicated outside of their original settings. ${ }^{9}$ Without improving the quality and reporting of IR for global health by systematically and appropriately documenting context across different settings, we will fail in our efforts to bring EBP to scale to achieve universal health coverage.

\section{GAPS WITHIN EXISTING GUIDELINES FOR IR IN ADDRESSING CONTEXT IN LMIC SETTINGS}

We propose that the paucity of robust evidence-based ethical guidelines available for research ethics committees and implementation researchers is a factor that may help to explain the shortfall of high-quality IR in LMICs. In particular, existing guidelines do not differentiate between the ethics of IR and clinical researchthese are different. ${ }^{3}$ Ethical guidelines also do nothing to acknowledge that implementation strategies are the interventions that deliver the EBP within IR (in other words, these strategies are the intervention(s)), and that these are context-dependent. As an example, The International Ethical Guidelines for Health-related Research Involving Humans were updated in 2016 to include a small paragraph on IR that was within guidance for cluster randomised trials. ${ }^{13}$ These guidelines acknowledge that 'cluster randomised trials investigate interventions that have been proven to be effective elsewhere and this is termed implementation research'. The guidelines then state 'research ethics committees have the responsibility to determine whether the proposed research is ethically acceptable when the methodology calls for withholding an established effective treatment from the control arm'. In late 2019, the WHO Alliance for Health Policy and 
Systems Research and the WHO Global Health Ethics Unit published updated guidance on Ethical Considerations for Health Policy and Systems Research that state even if a health intervention is known to be effective in one setting, clinical equipoise may nonetheless support evaluation of its effectiveness or implementation in another setting, for which evidence is lacking. ${ }^{14}$ Such ethical guidelines require expansion to address issues specifically pertaining to IR questions, which are not identical to those pertaining to clinical research questions within LMICs settings.

The Special Programme for Research and Training in Tropical Diseases (TDR) and the WHO improved on these guidelines by publishing training materials on IR and identifying associated ethical considerations. ${ }^{3}$ These materials suggest that equipoise may no longer lie in the clinical effectiveness of a trialed intervention, but in how precisely to implement the intervention in the new context in which the study will be conducted. These training materials are particularly useful as they include important case examples that allow participants to apply the theory on ethical standards for IR in practice.

\section{CONTEXTUAL EQUIPOISE FOR IR IN RESOURCE-P0OR SETTINGS}

To facilitate improvements in both ethical guidelines for IR and the evaluation and reporting of context in relation to IR in LMICs, we propose that there is a need to distinguish between equipoise for clinical interventions and equipoise for implementation strategies to deliver the EBP. Whereas clinical research that tests the effectiveness of a novel treatment/intervention using an RCT must uphold the ethical principle of clinical equipoise (ie, genuine uncertainty within the expert medical community about the efficacy or effectiveness of the preferred treatment ${ }^{15}$ ), this is no longer applicable for IR that uses an RCT to test the effectiveness of the implementation strategies to deliver care that is known to be clinically effective in a different context. ${ }^{16}$ Instead, a different ethical concern becomes salient regarding the level of uncertainty about the contextual conditions under which implementation will be effective. In such cases, we argue that the new ethical paradigm of contextual equipoise (ie, 'genuine uncertainty as to whether the implementation strategies will effectively deliver the EBP in a new context'), emerges as relevant. ${ }^{16}$

While a consideration of context is clearly of relevance to both clinical and implementation trials, typically seen with embedded process evaluations that investigate context-mechanism-outcome interactions, ${ }^{17}$ the notion of contextual equipoise is particularly pertinent for implementation trials. This is because such trials are specifically designed on the basis that there is uncertainty in how different implementation strategies will function within different contexts. Qualitative and quantitative methods documenting how context directly influences the effectiveness of implementation strategies, as well as mechanisms of action in delivering a specific clinical intervention across multiple contexts, can help to conceptualise a theory or model detailing the contexts and conditions where a given implementation strategy is effective. Over time, evidence accumulation will provide insight into strategies that require more contextual adaptation for maximal effectiveness, and strategies that may be more 'transferable' without as much adaptation.

\section{PROPOSED METHODS FOR EVALUATING THE INFLUENCE OF CONTEXT ON IMPLEMENTATION STRATEGIES}

Inadequate reporting of context is a well-known phenomenon in health studies. ${ }^{18}$ The difficulty in adequately addressing and reporting context may be due to the fact that the way in which it influences implementation efforts is a complex phenomenon operating through multiple pathways, feedback loops and involving key stakeholders. ${ }^{19}$ Context may also emerge through implementation, and may be best understood through the actions of the actors involved in intervention delivery. ${ }^{19}$ To address the difficulties in addressing context to understand how to adapt the intervention to the new setting, as well as to conceptualise whether contextual equipoise is sustained, we propose an approach to help systematically identify contextual barriers and enablers that influence both the implementation strategies to deliver the EBP, as well as the mechanisms introduced by the implementation strategies to deliver the EBP. We also describe the importance of participatory research with community members in understanding the local context. Examples of relevant methods are described and summarised in box 1 .

\section{IMPLEMENTATION SCIENCE AND MIDDLE-RANGE THEORIES}

Whereas IR seeks to understand what, why and how the implementation strategies to implement EBP work in real-world settings, ${ }^{4}$ implementation science offers robust

Box 1 Methodologies to help with the design and evaluation of implementation research

Determinant frameworks: a theoretical framework that identifies contextual barriers and/or enables that are known to impact on the effectiveness of implementation efforts. ${ }^{21}$

- Theoretical frameworks: identifies determinants of behaviours that are known to influence implementation outcomes. ${ }^{32}$

- Implementation theories/middle-range theories: describes the mechanisms behind how a proposed intervention works. These theories can also be used to identify barriers and/or enablers to change and what needs to change. ${ }^{21}$

- Realist evaluation: evaluates how, and for whom, to what extent, and in what contexts a programme might 'work'. ${ }^{23}$ This understanding of how the context shapes the mechanisms that lead to outcomes can be expressed as a context-mechanism-outcome configuration. ${ }^{23}$

- Theory of change: a participatory theory driven approach to programme design and evaluation whose underlying principle is to improve our understanding of how and why a programme works. ${ }^{33}$ 
Table 1 Examples of how implementation science determinant frameworks can be applied to identify contextual determinants that influence implementation effectiveness of evidence-based practice

\section{Implementation framework Framework description}

Consolidated Framework for The CFIR includes five domains Implementation Research (CFIR)

$\begin{array}{ll}\text { Theoretical Domains } & \begin{array}{l}\text { The implementation of evidence- } \\ \text { bramework (TDF) }\end{array} \\ \text { on changing multiple behaviours } \\ \text { of different people } \\ \text { the use of theory to inform } \\ \text { behaviour change interventions } \\ \text { has been shown to improve the } \\ \text { implementation effectiveness. } \\ \text { The TDF represents a synthesis of } \\ \text { 128 determinants of behavioural } \\ \text { change. }\end{array}$

The Context and Implementation of Complex Interventions $(\mathrm{ClCl})$ framework

EBP, evidence-based practice; LMICs, low/middle-income countries.

The $\mathrm{CICl}$ framework is both a determinant and evaluation framework that contains seven geographical, epidemiological, sociocultural, socioeconomic, ethical, legal, political) that are known to influence the effectiveness of the methods to deliver $\mathrm{EBP}^{36}$

\section{Example determinant in the literature}

Opinion leaders: individuals Excluding religious leaders in an organisation who have a formal or informal influence on the attitudes and beliefs of their colleagues concerning the implementation of the intervention. ${ }^{34}$ from a community that is highly religious, may find issues with the acceptability of Human Papilloma Virus (HPV) vaccination in Mozambique. This arises with programmes that are context sensitive and need to be supported and publicly advocated by local religious leaders. ${ }^{35}$ external contextual domains (ie,

\section{Social influences (those} interpersonal processes that can cause individuals to change their thoughts, feelings or behaviours). ${ }^{32}$
Sociocultural: behaviour patterns surrounding the core of culture including historically derived and selected ideas, and values that are shared among members of a group. ${ }^{36}$
A systematic review on access barriers to, and facilitators of, voluntary medical male circumcision to prevent HIV transmission found that male circumcision negatively perceived as being practiced by other or foreign cultures and religions was a major barrier. ${ }^{37}$

A qualitative synthesis using interviews with key stakeholders assessed for barriers in implementing evidence-based mental healthcare into primary healthcare in six LMICs. ${ }^{40}$ Findings suggest stigma associated with a mental health diagnosis was a significant barrier in implementation. theoretically driven methodologies to carry out this research. ${ }^{20}$ In contrast to the methods applied to measure clinical effectiveness/efficacy, implementation science offers methods that have been specifically developed for IR such as theories, models, frameworks. ${ }^{21}$ While these theoretical models and frameworks are numerous, many place context as a core concept for understanding implementation and therefore will be instrumental in helping to determine contextual equipoise for the delivery and effectiveness of EBP in a new setting.

Implementation science theoretical determinant frameworks were developed to help researchers identify and account for specific contextual influences on the implementation of EBP. ${ }^{21}$ Determinant frameworks therefore offer a means for synthesising evidence on context that can then be used to help understand if any adaptations are needed and whether contextual equipoise is sustained to a degree that justifies the use of a control arm. Table 1 presents examples of how different determinant frameworks can be applied to identify important contextual determinants to implementation effectiveness.

Whereas determinant frameworks help to identify contextual barriers that influence the effect of implementation strategies on implementation outcomes, broad implementation theories and narrower in scope middle-range theories (ie, a way of connecting high-level social theory with empirically observable patterns ${ }^{22}$ ) help conceptualise how context influences the mechanisms through which implementation strategies bring about change. Such mechanisms include behavioural activation, empowerment and augmentation of organisational readiness to implement EBP (among others). The use of theory to investigate how contextual determinants interact with mechanisms introduced by the implementation strategies can therefore help unravel some of the complexities surrounding contextual equipoise, in particular for considering how such interactions are likely to be activated in the new setting. ${ }^{21}$ As an example, a 'realist evaluation' can build a middle-range theory that can be 
applied to components of an intervention where context is thought to be most relevant and where greatest uncertainty exists. Such an approach would help decompose how, and for whom, to what extent and in what contexts an intervention might 'work', ${ }^{23} 24$ expressed as 'contextmechanism-outcome' configurations. ${ }^{23}$

\section{'THEORY OF CHANGE' METHODOLOGY}

Although determinant frameworks and implementation and middle-range theories provide invaluable resources for helping to assess contextual equipoise, context as a concept has been subject to a diversity of definitions and uses, as well as a being seen as emergent through implementation. ${ }^{19}$ It is therefore likely that further uncertainty will remain in how the evidence on the influence of context will actually translate to a new setting. ${ }^{19}$ This raises a question of how best to pragmatically resolve this issue to ensure that any planned IR is underpinned by the ethical principle of beneficence.

Theory of change (ToC) methodology is a participatory approach involving stakeholders that allows the articulation of the 'theory' of how a complex interventional programme will work in reality, describing the necessary interventions to bring about the change, as well as the assumptions inherent to the programme and importantly the context of implementation. ${ }^{25}$ A key premise of the ToC of relevance here is that engaging stakeholders including patients and their carers in participatory planning for IR, is critical to develop a programme that is contextually appropriate. ${ }^{26}$ Participatory ToC workshops are essential as they can help to resolve the uncertainty surrounding contextual equipoise by placing the decision in the hands of those the intervention is intended to benefit.

\section{APPROACH TO CONCEPTUALISE WHETHER CONTEXTUAL EQUIPOISE IS SUSTAINED}

We describe three complementary methodologies that apply implementation science determinant frameworks, implementation/middle-range theories and participatory ToC workshops, to understand whether contextual equipoise exists-as follows:

1. A thorough review of the literature, to identify contextual barriers and enablers that influence either implementation strategies used to deliver the proposed EBP or the mechanisms introduced by the implementation strategies to deliver EBP. Implementation science determinant frameworks and implementation and middle-range theories can be used to guide such literature reviews.

2. In the pre-implementation phase of research, methods such as situation analyses, interviews with key stakeholders, ethnographic observations ${ }^{27} 28$ and more recently linguistic ethnographic observations, ${ }^{29}{ }^{30}$ can be guided by determinant frameworks to identify key contextual barriers that influence the delivery of EBP. The same methods can also be guided by implementation and middle-range theories to evaluate how context influences the mechanisms introduced by current implementation strategies to deliver EBP. Such methods have the potential to empirically expose relationships between contextual determinants that are locally relevant or context-implementation relationships that were not revealed in other settings.

These types of insight will directly inform the relevance of literature review evidence on context, the content of determinant frameworks for the new setting, and middle-range theories that incorporate context as a concept for understanding implementation. In doing so, different contextual features may be brought to the foreground or revealed as less relevant at the point of delivery. For example, the Practical Approach to Care Kit Child (a guide designed to equip clinicians to diagnose and manage common childhood conditions at the primary care level) process evaluation study revealed how primary care facilities in the Western Cape of South Africa demonstrated an institutionalised orientation to acute, episodic risk minimisation for children under 5 years, upheld by provincial documentation. ${ }^{31}$ The use of direct observations of clinical consultations was critical in revealing these contextual conditions which were not previously understood or discussed in published literature.

3. Participatory methods, such as ToC workshops, with relevant stakeholders can be used to review similarities and differences in contextual determinants for delivering EBP between the literature review and the evaluation of the local context. Such a process can be used to come to an agreement as to the most salient implementation strategies. ToCs can also involve community members in a collective decision-making process to determine the most appropriate implementation metrics and study designs to apply (including but not limited to implementation RCTs), which increases the likelihood of longer-term sustainability of the evaluated intervention. Table 2 provides details of how implementation determinant frameworks, middle-range theories and participatory ToC workshops can be applied to help determine whether contextual equipoise exists.

\section{PROPOSED OPERATIONAL DEFINITION OF CONTEXTUAL EQUIPOISE FOR IMPLEMENTATION TRIALS IN LMICS}

In light of our analysis, we propose that using a robust theory-driven approach to address context will help to improve both the quality of IR in LMICs and surrounding ethical issues. To help achieve this, we recommend an operational definition whereby contextual equipoise is sustained and an RCT is only used when there is uncertainty regarding the influence of context on the effectiveness of the implementation strategies used to deliver EBP (box 2), and/or regarding the mechanisms mobilised by the implementation strategies to bring about change (box 3). 
Table 2 Details of how implementation science and other theory-driven approaches can address ethical challenges for trials justified on contextual equipoise to inform sustainable policy-making in resource-poor settings

\begin{tabular}{lll}
\hline Recommendation & Implementation science methodology & Expected outcome \\
\hline $\begin{array}{ll}\text { Literature review of barriers and facilitators to } \\
\text { implementing EBP. }\end{array}$ & $\begin{array}{l}\text { Literature reviews guided by relevant IS } \\
\text { determinant frameworks to synthesise } \\
\text { evidence for the influence of context on the } \\
\text { effectiveness of the implementation strategies }\end{array}$ & $\begin{array}{l}\text { A synthesis of barriers and facilitators that } \\
\text { are known to influence the effectiveness }\end{array}$ \\
& $\begin{array}{l}\text { on implementation strategies and/ } \\
\text { A realist review of relevant literature to } \\
\text { imderstand the influence of context on the }\end{array}$ & implementation strategies in delivering EBP. \\
& mechanisms introduced by the methods used \\
& to delivery EBP, on key outcomes.
\end{tabular}

Evaluation of the local context to identify barriers and/enablers and relevant implementation strategies to implement the proposed EBP.

Participatory methods to compare findings from the evaluation of the local context and literature review to select appropriate implementation strategies and study design.

Use of determinant frameworks, to identify relevant barriers and/or enablers that influence implementation strategies to deliver EBP. Realist evaluation to understand how the context influences the mechanisms introduced by current methods/ implementation strategies to deliver EBP. Methods are available that can help to select appropriate implementation strategies including a tool developed by The Expert Recommendations for Implementing Change (ERIC) study. ${ }^{5}$ The ERIC tool offers a compilation of implementation strategies that are known to be effective in addressing specific contextual barriers.

\section{Relevant stakeholders in the participatory} theory of change (ToC) workshops, can review similarities and differences in contextual determinants for delivering EBP between the literature review and the evaluation of the local context and come to an agreement as to the most salient implementation strategies identified using the ERIC tool.

Participatory ToC workshops can also be used to determine if contextual equipoise exists and an RCT ethically justifiable for the set of implementation strategies they selected to deliver the EBP.

Effectiveness-implementation hybrid trials
Helps investigator select a study design that can be applied to address contextual equipoise with implementation research. ${ }^{41}$ There are three types of hybrid trials (ie, type 1 , type 2 and type 3 ), where the type of trial design selected depends on the availability of evidence for both the clinical components of the intervention as well as the implementation strategies for a given context.

A hybrid type 1 trial is usually a cluster randomised trial as only strong indirect evidence exists for the effectiveneness of the EBB in the local context.

A hybrid type 2 trial can be randomised or quasi-experimental and is appropriate when there is strong indirect evidence as to the influence of context on the implementation strategies and EBP in the local context. A hybrid type 3 trial is usually a quasiexperimental design and is appropriate when there is generalisable evidence as to the influence of context on the effectiveness of implementation strategies and the EBP in the local context.
A synthesis of contextual determinants that can influence the effectiveness of the implementation strategies and/or mechanisms introduced by the implementation strategies in deliver EBP.

A list of potential implementation strategies that are known to be effective overcoming identified contextual barriers.

Implementation strategies that are deemed to be acceptable and appropriate by the local community.

Selection of appropriate study design that is determined by existing evidence on relevant contextual barriers.

Selection of an appropriate study design that is based on existing evidence base.

EBP, evidence-based practice; RCT, randomised controlled trial. 
Box 2 Examples of implementation trials where contextual equipoise is sustained due to the uncertainty surrounding the influence of the local context on the effectiveness of methods to implement evidence-based practice

- A stepped-wedge cluster randomised trial evaluating the effectiveness of a novel vital sign device in detecting pre-eclampsia and shock to improve maternal mortality or morbidity as well as implementation effectiveness in 10 countries across Africa, India and Haiti. ${ }^{42}$ The sensitivity and positive predictive value of the device had been previously established in low-resource settings ${ }^{43}$ but it was unknown whether the device would be effective when implemented using lay healthcare workers with minimal training. In this instance, contextual equipoise is sustained as it was unknown whether the local context would influence the ability of lay health workers to use the vital sign device to accurately detect pre-eclampsia and shock.

- A cluster randomised controlled trial evaluated the effectiveness of a stepped-care intervention using lay health workers on common mental disorders in Zimbabwe. ${ }^{44}$ Contextual equipoise was sustained as previous effective cluster randomised trials using steppedcare provided by lay health workers, had been done in countries with better healthcare resources than in sub-Saharan Africa, including clinics where routine care was provided by physicians. In this instance, it was unknown whether a context where there may be different factors such as primary care workers awareness of mental health conditions, may influence the effectiveness of the lay health worker in implementing the stepped-care intervention.

Crucially, the findings from the analysis used to evaluate context are used to help elucidate the adaptations that are required for the intervention to be implemented in the new setting. This can help to ensure that the intervention responds to the needs of the health system. One can anticipate that an RCT may be appropriate when the contextual analysis reveals that several additional implementation strategies are required to deliver the EBP, whereas scale-up that includes the ongoing monitoring of the influence of context on the implementation strategies is required when only a few adaptations are required.

Although there will always be a degree of uncertainty around how the intervention should be adapted and whether contextual equipoise exists, we emphasise that the ultimate decision needs to be driven by the relevant stakeholders including patients and their carers within LMICs. We hope that providing a definition for contextual equipoise and emphasising the importance of evaluating and reporting the influence of context on implementation strategies to deliver EBP, will help to improve our ability to generalise and replicate findings outside of their original settings.

The training materials provided by the WHO/TDR on the ethics of IR have provided an important step forward in raising awareness of ethical issues associated with IR including ensuring the research is methodologically sound and RCTs are not inappropriately used. ${ }^{3}$ We build on these training materials to propose a definition of contextual equipoise and methods to investigate it to
Box 3 Examples of when contextual equipoise is sustained due to the uncertainty surrounding the influence of context on the mechanisms introduced by the intervention to produce improvement

- There have been several cluster randomised trials evaluating the effect of women's groups using participatory learning and action (PLA) to improve the delivery essential newborn care practices, on neonatal mortality. ${ }^{45}$ One of the proposed mechanisms by which this complex intervention is theorised to achieve improved neonatal survival, is through PLA empowering women to be able to improve their own health in the antenatal, delivery and postnatal period through the improved uptake of evidence-based practice. For example, where gender inequity constrains improvements in maternal survival, empowered groups could give women the understanding, confidence and support to seek care or advice outside of their homes. In this instance, contextual equipoise would be sustained for any subsequent trial where there was uncertainty as to whether something in the local context could act as a barrier to directly influence the mechanism of empowering women to take control of their own health needs.

- A study protocol for a randomised controlled, non-inferiority trial aims to test the effectiveness of using general health workers to deliver mental healthcare in primary healthcare settings, compared with specialist medical care delivered by psychiatric nurses for patients with severe mental illness. ${ }^{46}$ Uncertainty exists as to whether stigma will be greater at an integrated service that is in a person's locality, compared with a separate psychiatric clinical further from the place of residence. Contextual equipoise is therefore sustained as patients may experience increased stigma when receiving care closer to their home, which could directly influence the mechanisms introduced by the stepped-care programme in improving mental illness.

complement that of clinical equipoise, which is far better developed conceptually. Given the lack of publications using robust methodology for IR, guidance is needed to inform research ethics committees, on both the relevant ethical issues to consider as well as high-quality methodological approaches. Ethical guidelines specifically developed for IR could help to achieve this objective by setting international standards from both a methodological and ethical perspective. However, existence of guidelines alone will not necessarily ensure that research ethics committees adhere to recommendations, where including ethical guidelines in the accreditation of ethics committees may help to overcome this issue. There could also be efforts to develop tailored training programmes for REC members to orientate them to the complexities and how they can make judgements on contextual equipoise.

Ensuring methodological and therefore ethical standards for IR in LMICs are guided by the same high standards used in high-income countries will strengthen the trust in, and value of, IR within the global health community and allow IR to deliver UHC with high-quality care.

\section{Author affiliations}

${ }^{1}$ Centre for Implementation Science, Department of Health Service and Population Research, King's College London, London, UK 
${ }^{2}$ Institute of Psychiatry, Psychology and Neuroscience, Centre for Global Mental Health, King's College London, London, UK

${ }^{3}$ Centre for Innovative Drug Development and Therapeutic Trials for Africa (CDTAfrica), College of Health Sciences, Addis Ababa University, Addis Ababa, Ethiopia ${ }^{4}$ Health Service and Population Research Department, King's College London, London, UK

${ }^{5}$ Norwich Medical School, Faculty of Medicine and Health Sciences, University of East Anglia Faculty of Medicine and Health Sciences, Norwich, UK

${ }^{6}$ Institute of Global Health, University College London, London, UK

${ }^{7}$ King's College London, London, UK

Twitter Nadine Seward @nadineseward, Martin James Prince @MartinKGHI and Sridhar Venkatapuram @sridhartweet

Acknowledgements We would like to thank Shalini Ahuja and Leila Younes for reviewing the manuscript and providing insight as to the salience of this topic for implementation researchers in Global Health.

Contributors NadineS drafted the paper; NadineS and $\mathrm{CH}$ conceptualised the idea for the paper; CH, TC and NickS reviewed several drafts of the manuscript; SV reviewed the manuscript and offered expertise on the ethical aspects of equipoise; JM reviewed the manuscript and offered expertise for the evaluation of context; MP offered critical inputs and reviewed later drafts of the manuscript NickS provided technical expertise to the role of implementation science in contextual equipoise; all authors contributed to the evolution of the concept of contextual equipoise detailed in this paper.

Funding NadineS, CH, MJP and NickS are funded by the National Institute of Health Research (NIHR) Global Health Research Unit on Health System Strengthening in sub-Saharan Africa, King's College London (GHRU 16/136/54) using UK aid from the UK Government to support global health research. NickSs' research is further supported by the NIHR Applied Research Collaboration South London at King's College Hospital NHS Foundation Trust, and by the ASPIRES research programme in LMICs (Antibiotic use across Surgical PathwaysInvestigating, Redesigning and Evaluating Systems), funded by the Economic and Social Research Council. NickS is a member of King's Improvement Science, which offers cofunding to the NIHR ARC South London and comprises a specialist team of improvement scientists and senior researchers based at King's College London. Its work is funded by King's Health Partners (Guy's and St Thomas' NHS Foundation Trust, King's College Hospital NHS Foundation Trust, King's College London and South London and Maudsley NHS Foundation Trust), Guy's and St Thomas' Charity and the Maudsley Charity. $\mathrm{CH}$ additionally receives funding support from AMARI as part of the DELTAS Africa Initiative (DEL-15-01). The views expressed here are not necessarily those of the NIHR or the Department of Health and Social Care, the NHS, the ESRC, AMARI or the DELTAS Africa Initiative.

Competing interests None declared.

Patient and public involvement Patients and/or the public were not involved in the design, or conduct, or reporting, or dissemination plans of this research.

Patient consent for publication Not required.

Provenance and peer review Not commissioned; externally peer reviewed.

Data availability statement There are no data in this work

Open access This is an open access article distributed in accordance with the Creative Commons Attribution Non Commercial (CC BY-NC 4.0) license, which permits others to distribute, remix, adapt, build upon this work non-commercially, and license their derivative works on different terms, provided the original work is properly cited, appropriate credit is given, any changes made indicated, and the use is non-commercial. See: http://creativecommons.org/licenses/by-nc/4.0/.

\section{ORCID iDs}

Nadine Seward http://orcid.org/0000-0002-4821-9437

Charlotte Hanlon http://orcid.org/0000-0002-7937-3226

Jamie Murdoch http://orcid.org/0000-0002-9021-3629

Tim Colbourn http://orcid.org/0000-0002-6917-6552

Martin James Prince http://orcid.org/0000-0003-1379-7146

Sridhar Venkatapuram http://orcid.org/0000-0003-3076-0783

\section{REFERENCES}

1 United Nations. Transforming our world: the 2030 agenda for sustainable development, 2015. Available: http://www.un.org/ga/ search/view_doc.asp?symbol=A/RES/70/1\&Lang=E
2 Peterson HB, Haidar J, Fixsen D, et al. Implementing innovations in global women's, children's, and adolescents' health: Realizing the potential for implementation science. Obstet Gynecol 2018;131:423-30.

3 World Health Organization. Ethics in implementation research. Facilitator's guide. Geneva: World Health Organization, 2019. Available: https://apps.who.int/iris/bitstream/handle/10665/325608/ 9789241515375-eng.pdf?ua=1 [Accessed Dec 2019].

4 Peters DH, Adam T, Alonge O, et al. Implementation research: what it is and how to do it. BMJ 2013;347:f6753.

5 Powell B, Thomas W, Matthew C. A refined compilation of implementation strategies: results from the expert recommendations for implementing change (ERIC) project. 10, 2015.

6 Waltz TJ, Powell BJ, Fernández ME, et al. Choosing implementation strategies to address contextual barriers: diversity in recommendations and future directions. Implement Sci 2019;14:42.

7 Ridde V, Pérez D, Robert E. Using implementation science theories and frameworks in global health. BMJ Glob Health 2020;5:e002269.

8 Ridde $\mathrm{V}$. Need for more and better implementation science in global health. BMJ Glob Health 2016;1:e000115.

9 Alonge $\mathrm{O}$, Rodriguez DC, Brandes N, et al. How is implementation research applied to advance health in low-income and middleincome countries? BMJ Glob Health 2019;4:e001257.

10 Theobald S, Brandes N, Gyapong M, et al. Implementation research: new imperatives and opportunities in global health. Lancet 2018;392:2214-28.

11 Yapa HM, Bärnighausen T. Implementation science in resource-poor countries and communities. Implement Sci 2018;13:154.

12 Pantoja T, Opiyo N, Lewin S, et al. Implementation strategies for health systems in low-income countries: an overview of systematic reviews. Cochrane Database Syst Rev 2017;9:CD011086-CD.

13 CIOMS. Council for international organizations of medical sciences (CIOMS). International ethical guidelines for health-related research involving humans. Geneva, Switzerland: Council for International Organizations of Medical Sciences, 2016. https://cioms.ch/wpcontent/uploads/2017/01/WEB-CIOMS-EthicalGuidelines.pdf

14 World Health Organization. Ethical considerations for health policy and systems research, 2019. Available: https://apps.who.int/iris/ bitstream/handle/10665/330033/9789241516921-eng.pdf?ua=1 [Accessed Nov 2019].

15 Freedman B. Equipoise and the ethics of clinical research. $N$ Engl J Med 1987;317:141-5.

16 Gopichandran V, Luyckx VA, Biller-Andorno N, et al. Developing the ethics of implementation research in health. Implement Sci 2016;11:161.

17 Moore GF, Audrey S, Barker M, et al. Process evaluation of complex interventions: medical Research Council guidance. BMJ 2015;350:h1258.

18 Wells M, Williams B, Treweek S, et al. Intervention description is not enough: evidence from an in-depth multiple case study on the untold role and impact of context in randomised controlled trials of seven complex interventions. Trials 2012;13:95.

19 Meier N, Dopson S. Context in action and how to study it. Oxford Scholarship Online, 2019.

20 Eccles MP, Mittman BS. Welcome to implementation science. Implementation Science 2006;1.

21 Nilsen P. Making sense of implementation theories, models and frameworks. Implement Sci 2015;10:53.

22 Merton RK. On sociological theories of the middle range. on theoretical sociology. New York: Free Press, 1948: 39-72.

23 Pawson R, Tilley N. Realistic evaluation. London: Sage, 1997.

24 Pawson R, Sridharan S. Theory-driven evaluation of public health programmes. In: Killoran A, Kelly M, eds. Evidence-Based public health: effectiveness and efficacy. Oxford: Oxford University Press, 2010.

25 Vogel I. Review of the use of theory of change in international development. UK: Department for International Development, 2012.

26 Israel BA, Schulz AJ, Parker EA, et al. Review of community-based research: assessing partnership approaches to improve public health. Annu Rev Public Health 1998:19:173-202.

27 Hammersley M. Ethnography principles in practice. Routledge,London, 1992.

28 Morgan-Trimmer S, Wood F. Ethnographic methods for process evaluations of complex health behaviour interventions. Trials 2016;17:232.

29 Rampton BK MT, Barwell H, Creese RA, et al. Linguistic ethnography: a discussion paper coordinating Committee UK linguistic ethnography forum, 2004.

30 Murdoch J. Process evaluation for complex interventions in health services research: analysing context, text trajectories and disruptions. BMC Health Serv Res 2016;16:407. 
31 Murdoch JCR, Cornick R, Picken S. Addressing the quality and scope of paediatric primary care in South Africa: evaluating contextual impacts of the introduction of the practical approach to care kit for children (pack child), 2020.

32 Atkins L, Francis J, Islam R, et al. A guide to using the theoretical domains framework of behaviour change to investigate implementation problems. Implement Sci 2017:12:77.

33 Weiss C. Nothing as Practical as Good Theory: Exploring Theorybased Evaluation for Comprehensive Community Initiatives for Children and Families. In: JP C, ed. New approaches to evaluating community initiatives: concepts, methods, and contexts. Washington, DC: Aspen Institute, 1995.

34 Damschroder LJ, Aron DC, Keith RE, et al. Fostering implementation of health services research findings into practice: a consolidated framework for advancing implementation science. Implement Sci 2009;4:50

35 Soi C, Gimbel S, Chilundo B, et al. Human papillomavirus vaccine delivery in Mozambique: identification of implementation performance drivers using the consolidated framework for implementation research (CFIR). Implement Sci 2018;13:151.

36 Pfadenhauer LM, Gerhardus A, Mozygemba K, et al. Making sense of complexity in context and implementation: the context and implementation of complex interventions $(\mathrm{CICl})$ framework. Implement Sci 2017;12:21.

37 Carrasco MA, Wilkinson J, Kasdan B, et al. Systematic review of barriers and facilitators to voluntary medical male circumcision in priority countries and programmatic implications for service uptake. Glob Public Health 2019;14:91-111.

38 Grol R, Grimshaw J. From best evidence to best practice: effective implementation of change in patients' care. Lancet 2003;362:1225-30.
39 Noar SM, Zimmerman RS. Health behavior theory and cumulative knowledge regarding health behaviors: are we moving in the right direction? Health Educ Res 2005;20:275-90.

40 Petersen I, van Rensburg A, Kigozi F, et al. Scaling up integrated primary mental health in six low- and middle-income countries: obstacles, synergies and implications for systems reform. BJPsych Open 2019;5:e69.

41 Curran GM, Bauer M, Mittman B, et al. Effectiveness-implementation hybrid designs: combining elements of clinical effectiveness and implementation research to enhance public health impact. Med Care 2012;50:217-26.

42 Vousden N, Lawley E, Nathan HL, et al. Effect of a novel vital sign device on maternal mortality and morbidity in low-resource settings: a pragmatic, stepped-wedge, cluster-randomised controlled trial. Lancet Glob Health 2019;7:e347-56.

43 Nathan HL, de Greeff A, Hezelgrave NL, et al. Accuracy validation of the Microlife 3AS1-2 blood pressure device in a pregnant population with low blood pressure. Blood Press Monit 2015;20:299-302.

44 Chibanda D, Weiss HA, Verhey R, et al. Effect of a primary carebased psychological intervention on symptoms of common mental disorders in Zimbabwe: a randomized clinical trial. JAMA 2016;316:2618-26.

45 Prost A, Colbourn T, Seward N, et al. Women's groups practising participatory learning and action to improve maternal and newborn health in low-resource settings: a systematic review and metaanalysis. The Lancet 2013;381:1736-46.

46 Hanlon C, Alem A, Medhin G, et al. Task sharing for the care of severe mental disorders in a low-income country (TaSCS): study protocol for a randomised, controlled, non-inferiority trial. Trials 2016;17:76. 